\title{
Search for Non-Standard, Rare or Invisible Decays of the Higgs Boson with the ATLAS Detector
}

\author{
Paul Thompson*† \\ University of Birmingham \\ E-mail: pdtahep.ph.bham.ac.uk
}

\begin{abstract}
The search for non-standard, invisible or rare decays of the Higgs boson is an important area of investigation at the LHC because of the sensitivity to effects from physics beyond the Standard Model. In this article three recent measurements from the ATLAS Collaboration are presented; the search for Higgs boson decays to $Z \gamma$, the search for an invisibly decaying Higgs boson or dark matter candidate produced in association with a $Z$ boson, and the search for Higgs boson decays to $\phi \gamma$ and $\rho \gamma$.
\end{abstract}

EPS-HEP 2017, European Physical Society conference on High Energy Physics 5-12 July 2017

Venice, Italy

* Speaker.

${ }^{\dagger}$ On behalf of the ATLAS Collaboration 


\section{Introduction}

Following the observation [1, 2] of a Higgs boson, $H$, with a mass of approximately $125 \mathrm{GeV}$ [3] by the ATLAS [4] and CMS Collaborations, the properties of its interactions have been measured in subsequent publications. The couplings to Standard Model (SM) elementary particles were measured and confirmed to be consistent with the predictions for a SM Higgs boson within the present uncertainties [5]. The search for invisible or rare decays of the Higgs boson are an important area of investigation because any observation of them with the present LHC luminosity would be a clear signature of physics beyond the Standard Model (BSM). In this article three recent measurements from the ATLAS Collaboration are presented: the search for Higgs decays to $Z \gamma$ [6], the search for an invisibly decaying Higgs boson or dark matter candidates produced in association with a $Z$ boson [7], and the search for Higgs decays to $\phi \gamma$ and $\rho \gamma$ [8].

\section{Searches for the $Z \gamma$ decay mode of the Higgs boson}
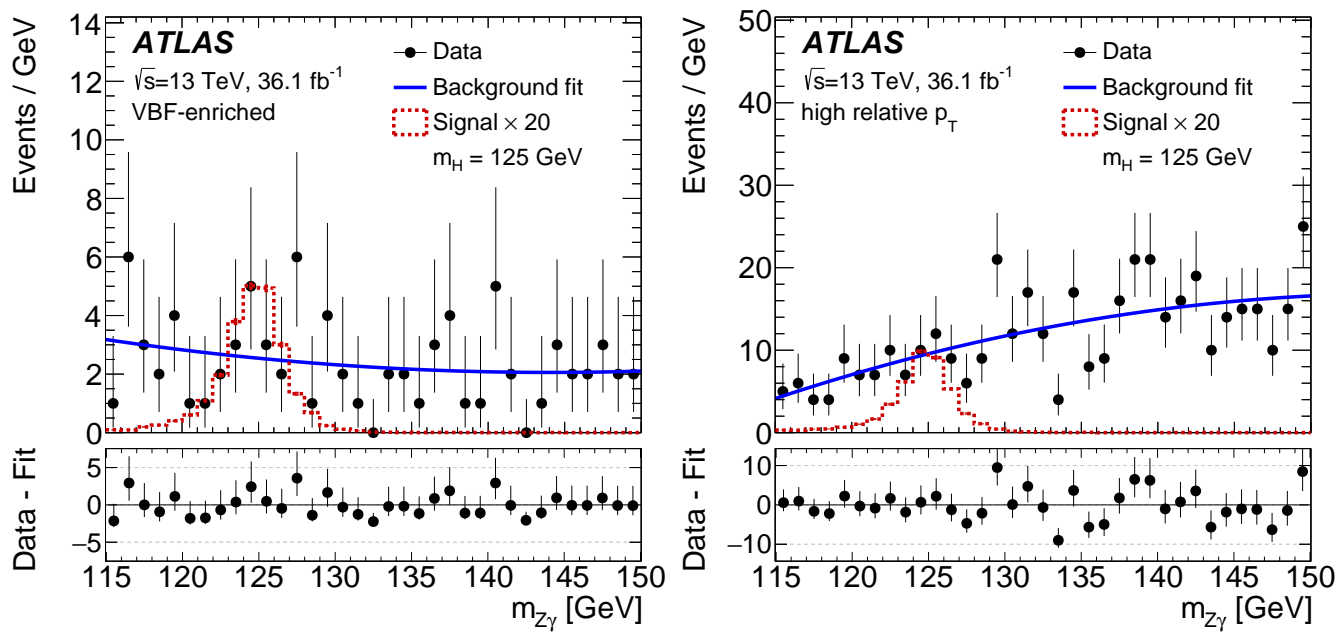

Figure 1: The $m_{Z \gamma}$ distributions of events satisfying the $H \rightarrow Z \gamma$ selection in data for two example event categories: (a) VBF-enriched, (b) high $p_{\mathrm{T}}^{\gamma}$. The points represent the data and the statistical uncertainty. The solid lines show the background-only fits to the data, performed independently in each category. The dashed histogram corresponds to the expected signal for a SM Higgs boson with $m_{H}=125 \mathrm{GeV}$ decaying to $Z \gamma$ with a rate 20 times the SM prediction. The bottom part of the figures shows the residuals of the data with respect to the background-only fit.

In the SM, the $Z \gamma$ decay proceeds through loop diagrams similar to the $H \rightarrow \gamma \gamma$ decay. The branching ratio for the Higgs boson decay to $Z \gamma$ is predicted by the SM to be $B(H \rightarrow Z \gamma)=$ $(1.54 \pm 0.09) \times 10^{-3}$ at $m_{H}=125.09 \mathrm{GeV}$. A $H \rightarrow Z \gamma$ branching ratio different from the SM prediction would be expected if, for example, the $H$ were a neutral scalar of different origin [9].

In the analysis events are split into mutually exclusive event categories that are optimised to improve the sensitivity of the search using the expected signal-to-background ratio and the expected three-body invariant mass resolution. The invariant $Z \gamma$ mass $\left(m_{Z \gamma}\right)$ for two of the most sensitive categories are shown in Figure 1. For $Z \rightarrow \mu \mu$ candidates the invariant mass resolution of the $Z$ boson candidate is improved by $3 \%$ when correcting the muon momenta for the collinear radiation 
of photons from final-state leptons. A constrained kinematic fit is applied to recompute the fourmomenta of the dilepton pair for $Z \rightarrow \mu \mu$ and $Z \rightarrow e e$ candidates. The fit models the lepton energy and momentum response as a Gaussian distribution for each lepton, and the Gaussian width is given by the expected resolution. The kinematic fit improves the $m_{\mu \mu \gamma}\left(m_{e e \gamma}\right)$ resolution by $7 \%$ (13\%) for $m_{H}=125 \mathrm{GeV}$.

No evidence of a localised excess is visible near the anticipated Higgs mass $m_{H}=125.09 \mathrm{GeV}$. The observed 95\% CL limit on $\sigma(p p \rightarrow H) \cdot B(H \rightarrow Z \gamma)$ is found to be 6.6 times the SM prediction, corresponding to the limit of $547 \mathrm{fb}$. Assuming SM Higgs boson production, the upper limit on the branching ratio of the Higgs boson decay to $Z \gamma$ is found to be $1.0 \%$. The expected 95\% CL limit on $\sigma(p p \rightarrow H) \cdot B(H \rightarrow Z \gamma)$ assuming no (a SM) Higgs boson decay to $Z \gamma$ is 4.4 (5.2) times the SM prediction.

\section{Search for an invisibly decaying Higgs boson in association with a $Z$ boson}


Figure 2: Observed $E_{\mathrm{T}}^{\text {miss }}$ distribution in the $e e$ (left) and $\mu \mu$ (right) channel compared to the signal and background predictions. The error band shows the total statistical and systematic uncertainty on the background prediction. The ratio plot gives the observed data yield over the background prediction (black points) as well as the signal-plus-background contribution divided by the background prediction (blue or purple line) in each $E_{\mathrm{T}}^{\text {miss }}$ bin. The $Z H \rightarrow \ell \ell+$ inv signal distribution is shown with $B_{H \rightarrow \text { inv }}=0.3$, which is the value most compatible with data. The simulated DM distribution with $m_{\text {med }}=500 \mathrm{GeV}$ and $m_{\chi}=100 \mathrm{GeV}$ is also scaled (with a factor of 0.27 ) to the best-fit contribution.

In the SM, the invisible decay of the Higgs boson $(H \rightarrow Z Z \rightarrow v v v v)$ has a branching ratio $B_{H \rightarrow \text { inv }}$ of $1.06 \times 10^{-3}$ for $m_{H}=125 \mathrm{GeV}$ [10]. A larger $B_{H \rightarrow \text { inv }}$ can exist in many extensions of the SM. For example, a Higgs boson can decay to dark matter (DM) through the Higgs portal model $[11,12]$. Observation of a $B_{H \rightarrow \text { inv }}$ significantly above the SM value would give a strong indication for BSM physics.

This search is carried out in a $\ell \ell+E_{\mathrm{T}}^{\text {miss }}$ final state, which contains large $E_{\mathrm{T}}^{\text {miss }}$ and a pair of high- $p_{\mathrm{T}}$ isolated electrons $(e e)$ or muons $(\mu \mu)$. Backgrounds are reduced by removing events with extra leptons or any jets containing $b$-hadrons (" $b$-jets") and by requiring a boosted $Z$ boson which is back to back with the missing transverse momentum vector $\left(\vec{E}_{\mathrm{T}}^{\text {miss }}\right)$. No significant excess over the SM background expectation is observed for the $E_{\mathrm{T}}^{\text {miss }}$ data distributions (see Figure 2) and a 
95\% CL upper limit is placed on $B_{H \rightarrow \text { inv }}$, assuming the SM prediction for the $Z H$ production crosssection. Using the combined $e e$ and $\mu \mu$ channel, the observed and expected limits on $B_{H \rightarrow \text { inv }}$ are $67 \%$ and $39 \%$, respectively. The data is used to constrain the mediator and WIMP masses of dark matter models.

\section{Search for exclusive Higgs boson decays to $\phi \gamma$ and $\rho \gamma$}
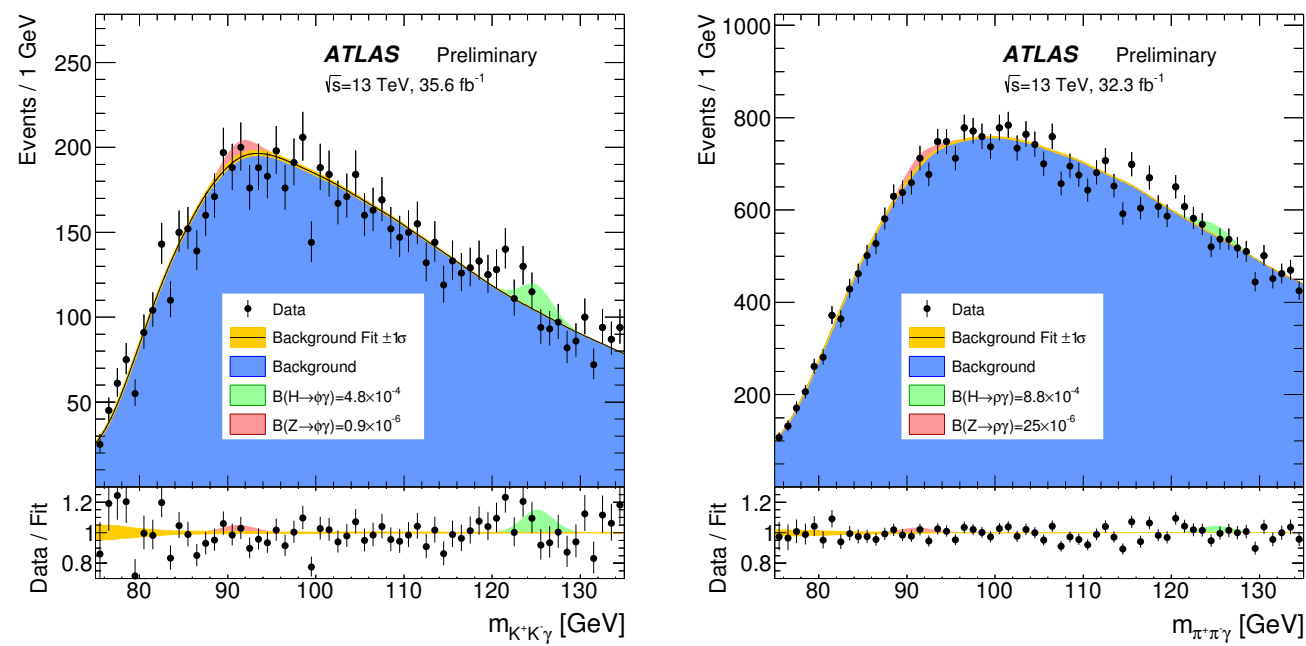

Figure 3: The $m_{K^{+} K^{-} \gamma}$ and $m_{\pi^{+} \pi^{-}}$distributions of the selected $\phi \gamma$ and $\rho \gamma$ candidates, respectively, along with the results of the maximum-likelihood fit with a background-only model. The Higgs and $Z$ boson contributions for the branching fraction values corresponding to the observed $95 \%$ CL upper limits are also shown. Below the figures the ratio of the data and the background only fit is shown.

In the Standard Model (SM) the interactions to fermions are implemented through Yukawa interactions, and a wealth of beyond-the-SM theories predict substantial modifications of the relevant Higgs boson couplings to fermions. An overview of relevant models of new physics is provided in Ref. [10]. The Higgs boson decay to a $\phi$ or $\rho(770)$ meson and a photon provides sensitivity to its couplings to the strange-quark, and the up- and down-quarks, respectively. The expected SM branching fractions are $B(H \rightarrow \phi \gamma)=(2.31 \pm 0.11) \times 10^{-6}$ and $B(H \rightarrow \rho \gamma)=(1.68 \pm 0.08) \times$ $10^{-5}[13]$.

The transverse momentum of the $M$ candidates (where $M$ is either the $\phi$ or $\rho$ meson) is required to be greater than a threshold that varies as a function of the invariant mass of the three-body system, $m_{M \gamma}$. The background processes are modelled with a non-parametric data-driven approach using templates to describe the kinematic distributions and the background normalisation and shape are simultaneously extracted from a fit to the data.

The results of the background-only fits for the $\phi \gamma$ and $\rho \gamma$ analyses are shown in Figure 3. The observed yields are consistent with the number of events expected from the background only prediction within the systematic and statistical uncertainties. On the basis of the observed data, upper limits are set on the branching fractions for the Higgs and $Z$ boson decays to $M \gamma$. The observed 95\% CL upper limits on the branching fractions for $H \rightarrow \phi \gamma$ and $Z \rightarrow \phi \gamma$ decays are 208 and 87 times the expected SM branching fractions, respectively. The corresponding values for the $\rho \gamma$ decays are 52 and 597 times the expected SM branching fractions, respectively. 


\section{References}

[1] ATLAS Collaboration. Observation of a new particle in the search for the standard model Higgs boson with the ATLAS detector at the LHC. Phys. Lett. B, 716:1-29, 2012.

[2] CMS Collaboration. Observation of a new boson at a mass of $125 \mathrm{GeV}$ with the CMS experiment at the LHC. Phys. Lett. B, 716:30-61, 2012.

[3] ATLAS and CMS Collaborations. Combined Measurement of the Higgs Boson Mass in pp Collisions at $\sqrt{s}=7$ and $8 \mathrm{TeV}$ with the ATLAS and CMS Experiments. Phys. Rev. Lett., 114:191803, 2015.

[4] ATLAS Collaboration. The ATLAS experiment at the CERN Large Hadron Collider. JINST, 3:S08003, 2008

[5] ATLAS and CMS Collaborations. Measurements of the Higgs boson production and decay rates and constraints on its couplings from a combined ATLAS and CMS analysis of the LHC $p p$ collision data at $\sqrt{s}=7$ and $8 \mathrm{TeV}$. JHEP, 08:045, 2016.

[6] ATLAS Collaboration. Searches for the $Z \gamma$ decay mode of the Higgs boson and for new high-mass resonances in $p p$ collisions at $\sqrt{s}=13 \mathrm{TeV}$ with the ATLAS detector. arXiv, hep-ex:1708.00212, 2017. Submitted to JHEP.

[7] ATLAS Collaboration. Search for an invisibly decaying Higgs boson or dark matter candidates produced in association with a $Z$ boson in $p p$ collisions at $\sqrt{s}=13 \mathrm{TeV}$ with the ATLAS detector. arXiv, hep-ex:1708.09624, 2017. Submitted to Phys. Lett.

[8] ATLAS Collaboration. Search for exclusive Higgs and Z boson decays to $\phi \gamma$ and $\rho \gamma$ with the ATLAS Detector. ATLAS-CONF-2017-057, July 2017.

[9] Ian Low, Joseph Lykken, and Gabe Shaughnessy. Singlet scalars as Higgs imposters at the Large Hadron Collider. Phys. Rev. D, 84:035027, 2011.

[10] LHC Higgs Cross Section Working Group. Handbook of LHC Higgs Cross Sections: 4. Deciphering the Nature of the Higgs Sector. arXiv, hep-ph:1610.07922, 2016.

[11] M. C. Bento, O. Bertolami, R. Rosenfeld, and L. Teodoro. Self-interacting dark matter and the Higgs boson. Phys. Rev. D, 62:041302, Jul 2000.

[12] Christoph Englert, Tilman Plehn, Dirk Zerwas, and Peter M. Zerwas. Exploring the Higgs portal. Phys. Lett. B, 703:298-305, 2011.

[13] Matthias König and Matthias Neubert. Exclusive Radiative Higgs Decays as Probes of Light-Quark Yukawa Couplings. JHEP, 08:012, 2015. 